\title{
Objects Relationship Modeling for Improving Object Detection Using Bayesian Network Integration
}

\author{
Youn-Suk Song and Sung-Bae Cho \\ Dept. of Computer Science, Yonsei University \\ 134 Shinchon-dong, Sudaemun-ku, Seoul 120-749, Korea \\ corlary@sclab.yonsei.ac.kr, sbcho@cs.yonsei.ac.kr
}

\begin{abstract}
Object detection is very important to service robots. Many tasks for service such as delivery, cleaning, and health-care for elderly people are strongly related to objects. Conventional approaches for object detection are mainly based on the geometric models, because they have been applied to static environments. In indoor environments having uncertainty, they have limitation in some situations where interesting objects are occluded by other ones or small in the scene. Context information can be helpful to overcome these uncertain situations. In this paper, we adopt objects as context information to allow for service robots to predict the probability of interesting objects through observed ones. For this, an object relationship model based on Bayesian network (BN) and integration method are proposed. Experimental results confirm that the proposed method predicts the objects very well.
\end{abstract}

\section{Introduction}

The studies of object detection and scene understating using visual sensors for service robot are important. Conventional approaches mainly used in industrial environments mainly obtain the information from images based on predefined geometric models, so they have shown limitations in uncertain situations when objects are occluded by other ones or small [1]. Since the service robots reside in human environments such as home and office, it is very important to manage them. Knowledge-based approaches can be a good choice to manage these uncertain situations, because they can give robots context information to resolve the uncertainty. Bayesian network (BN) is well known as a good method to represent the degree of uncertainty of some facts in a probabilistic framework [2]. There are many relevant works as follows.

Table 1. Relevant works

\begin{tabular}{|c|c|c|c|}
\hline Researcher & Year & Context & Modeling method \\
\hline Socher & 2000 & Voice & Bayesian network \\
\hline Luo & 2003 & Location & Bayesian network \\
\hline Song & 2005 & Object & Bayesian network \\
\hline
\end{tabular}

In this paper, BN model is adopted to represent the relationship between objects and the probability of object being present for object detection for service robots. It can help service robots to detect objects. 


\section{Bayesian Network Modeling}

Bayesian network is the DAG (directed acyclic graph) model to evaluate the belief of variables using the dependency between them based on the Bayes' rule. The nodes represent random variables while the edges denote the dependencies of them. The two nodes linked by an edge have the joint probability distribution respectively, in which the parent has prior probability, and the child has the conditional probability in the context of parent's states.

In many applications, BN has shown a good performance, but it is also known that the modeling process of $\mathrm{BN}$ has difficulties in several perspectives such as complexity of modeling according to scalability of domain and eliciting parameters for conditional probability [2]. For this, in the mid 1990's, Laskey et al. proposed the modularized design method under object-oriented paradigm for modeling military situation. They proposed the approach to integrate pre-designed Bayesian networks for the situations by integration methods like noisy-min [3].

We propose a novel BN structure based on a common-cause structure, binary-cross conditional probability table (CPT) setting method, and the method integrating the basic BNs with high-level BN using virtual nodes for object relationship modeling.

\section{Object Relationship Modeling}

To make object relationship, it is required to define the criteria of their relationship. Activities can be used for explaining the purposes of objects existence. Activity theory supports this idea. Our modeling process is composed of two stages: object-stage and activity-stage. In object-stage, the basic relationships called primitive BNs are defined between objects. They are small relatively, so it is easy to make them. Next stage, they are integrated for extending the object relationships if it is needed for reasoning. Fig. 1 shows the overall process of modeling.
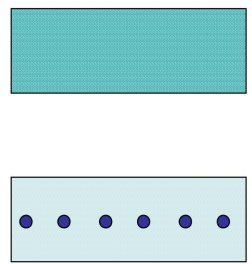

Defining Object
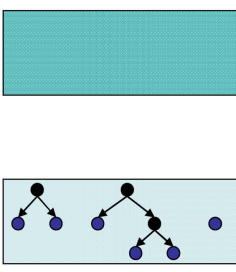

Object Modeling
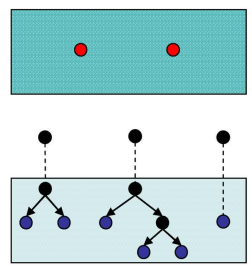

Defining Activities

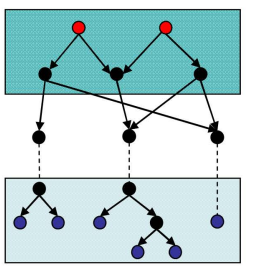

Integration
Activity Level

Virtual node

Object Leve

Activity

- Primitive

Activity

Activity stage

Fig. 1. Modeling Process

After explaining about the service robot and the domain in this section, the proposed $\mathrm{BN}$ model will be described. 


\subsection{Service Robot}

We construct the BNs in the environments of 15 places and 29 objects. The environments are summarized in Table 2.

Table 2. Service environment

\begin{tabular}{|c|l|}
\hline Category & \multicolumn{1}{c|}{ Contents } \\
\hline Places (15) & $\begin{array}{l}\text { Lecture room, Meeting room, Seminar room, Computer room, Prof. of } \\
\text { fice, Admin. office, Guard office, Lab., Hallway, Stair, Hall, Elevator, } \\
\text { Toilet, Former Toilet, Rest room }\end{array}$ \\
\hline Objects (29) & $\begin{array}{l}\text { Table, Side Chair, Castor Chair, Lectern, Cabinet, Bookcase, Garbage } \\
\text { can, Sink, Seat toilet, Wall clock, Air conditioner, Telephone, Desktop } \\
\text { PC, Notebook PC, Mouse, LCD Monitor, Keyboard, Beam Projector, } \\
\text { Projection Screen, Audio, Speaker, Microphone, Wall white board, Ca } \\
\text { stor white board, Partition, Curtain, Water bucket, Door, Window }\end{array}$ \\
\hline
\end{tabular}

The proposed $\mathrm{BN}$ models in this paper are used for predicting the probability of registered objects by the discovered ones. When the robot executes detection, he can decide whether it is needed to try further detection.

\subsection{Activity Theory}

Activity theory gives us theoretical background of our idea that uses activity as the criterion of defining objects relationship. Vygosky suggested the simple activity model 'subject-tool-object' in 1920s. It helps us to explain why the objects (tools) exist together, because they are used for the same purpose (activity). Activity also has the property that can be subsumed by other activities like goals. This property can be also used to make the relationship and promote us to use hierarchical structure (tree shape) for modeling.

\subsection{Object Stage: Primitive Bayesian Network}

The primitive Bayesian networks are tree structures that each node has one parent and binary state, and they are composed of four kinds of basic nodes: activity node $(A)$, class node $(C)$, object node $(O)$, and virtual node $(V)$. The summary for every node and the hierarchical relations are as follows.

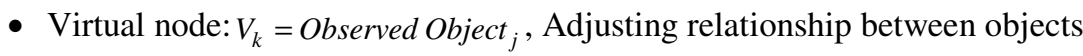

- Object node: $O_{j}$ can include $\left\{\right.$ Object $_{1}$, Object $_{2}, \ldots$ Object $_{l-1}$, Object $\left._{l}\right\} \cup\left\{V_{1}, V_{2}, \ldots V_{w-1}, V_{w}\right\}$, Representing observed objects or probability of target objects

- Class node: $C_{i}$ can include $\left\{C_{1}, C_{2}, \ldots, C_{n-1}, C_{n}\right\} \cup\left\{O_{1}, O_{2}, \ldots O_{m-1}, O_{m}\right\}$, Criterion of detailed object relationship

- Activity node: $A_{p}$ can include $=\left\{C_{1}, C_{2}, \ldots, C_{n-1}, C_{n}\right\} \cup\left\{O_{1}, O_{2}, \ldots O_{m-1}, O_{m}\right\}$, Root node, Criterion of primitive activity $\mathrm{BN}$ 
We define the relationship of objects as four types. They are described as follows:

- Equality: $P(X \mid Y)=P(Y \mid X)$

- Part-of: $P(Y \mid X)=P(X \mid Y)=1$

- Inequality: $P(X \mid Y) \neq P(Y \mid X)$ and $P(Z \mid X, Y) \neq P(Z \mid X), P(Z \mid Y)$

- Independence: $P(X \mid Y)=P(X)$ or $P(Y \mid X)=P(Y)$

Equality relationship means that influences between two objects are equal. Part-of relation is the special case of equality. This is the case one object is the part of other one like door and door handle. Inequality relationship is the case that two objects have different influence to each other. That is, one object is necessary condition for the other one. In general the objects that are used with many other objects are necessary condition for another one, because they have little information according to information theory. Independence relationship means that there is no influence between objects, so it is not under consideration. Structures mentioned above are shown in Fig. 2.

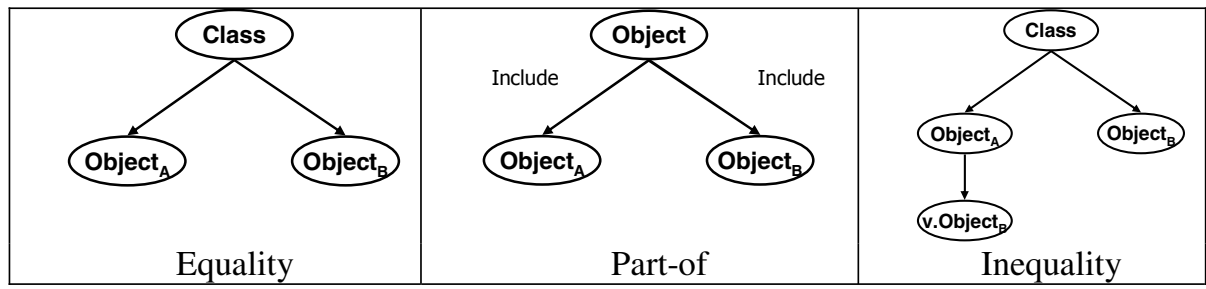

Fig. 2. Defined structures (v means virtual node)

We set the value of CPT of the BN model as follows.

$$
\begin{aligned}
& \sum_{\text {parent }_{i}} P\left(\text { child }_{j} \mid \text { parent }_{i}\right)=1\left(\text { child }_{\mathrm{j}} \text {, parent }{ }_{\mathrm{i}} \text { is the state of parent }\right) \\
& P\left(\text { child }_{\text {yes }} \mid \text { parent }_{y e s}\right)=P\left(\text { child }_{n o} \mid \text { parent }_{n p}\right)
\end{aligned}
$$

In this way, we can keep the probability distributions of all nodes uniformly after belief-updating without evidences. This is important for prediction. Next formula explains about this.

$$
\begin{aligned}
P\left(\text { Child }_{\text {yes }} \mid \text { Parent }\right)= & P\left(\text { Child }_{\text {yes }} \mid \text { Parent }_{\text {yes }}\right) P\left(\text { Parent }_{\text {yes }}\right) \\
& +P\left(\text { Child }_{\text {yes }} \mid \text { Parent }_{n o}\right) P\left(\text { Parent }_{n o}\right) \\
= & \alpha \times 0.5+(1-\alpha) \times 0.5 \\
= & 0.5\left(\text { we can use the same formula for } P\left(\text { Child }_{n o} \mid \text { Parent }\right)\right)
\end{aligned}
$$

Suppose $\alpha$ represent the probability of $P\left(\right.$ Child $_{y e s} \mid$ Parent $\left._{y e s}\right)$ and assign $1-\alpha$ to $P\left(\right.$ Child $_{\text {yes }} \mid$ Parent $\left._{n o}\right)$ (following to formula 1 ) then we can maintain the probability of all nodes as $(0.5,0.5)$ in the case that there are no evidences in the network having the uniform prior probability of activity node. This method allows us to just decide the 
value called as 'influence value' $P\left(\right.$ Child $_{\text {yes }} \mid$ Parent $\left._{\text {yes }}\right)$ to express the degree of relationship. If the influence value of child node has large one, it means it has large influence to other nodes belonged to the same class. This method is called binary-cross probability setting due to their formation when they are represented in the CPTs.

\subsection{Activity Stage: Integration}

If primitive BNs can be properly combined together, it becomes easier to extend and represent the object relationship. For this, we propose the method for integrating primitive BNs properly with high-level BNs (Activity BNs). This is efficient to make object relationship in the view point of reusability. Fig. 3 (left) shows the overall models.
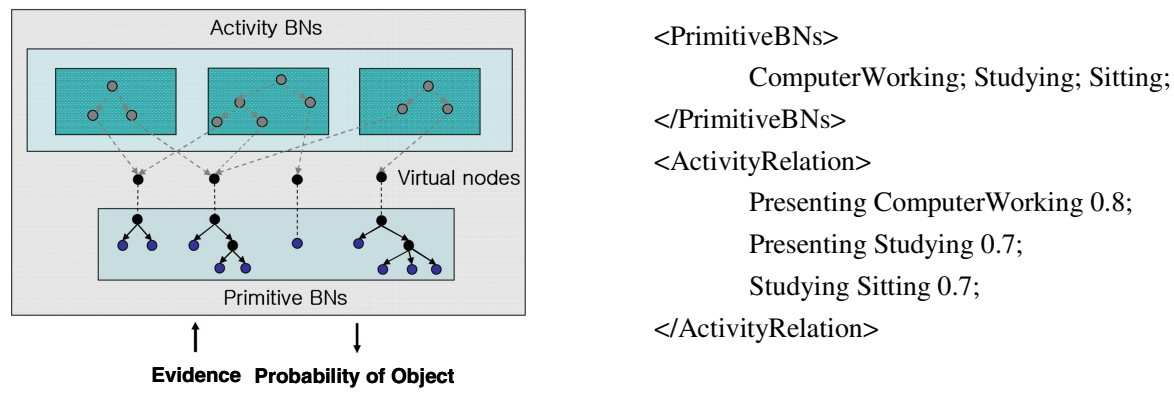

Fig. 3. Left: Overall models. Right: scripts for integration (presenting)

ActivityRelation describes the Activity BN structure. The values of root nodes of Primitive BNs are used for influence value of virtual nodes, the leaves of activity BNs. The process of inference is as follows.

1. Compute $P$ (root node $\mid$ Evidence) of primitive BNs

2. Construct activity BN following to the scripts in the Activity Relation

3. Make virtual nodes that have influence value computed in step 1 as the child of corresponding activity nodes in the activity BN

4. Set the evidence to virtual nodes and update the belief

5. Set the prior probability of primitive BNs with the new values of activity nodes. $\left(\right.$ Prior $_{\text {new }}=0.5+$ Activity $_{\text {activityBN }}-$ aof v.Activity ${ }_{\text {activityBN }}, a$ is influence value)

6. Inference the value of interesting object

\section{Experimental Results}

Experiments are carried out to verify the performance of the proposed BN model in five different places (Computer room, Laboratory, Rest room, Conference room, and 
Seminar room). We use BN model that represents the presenting-activity for detecting beam-projector existing in Conference room. It is designed by composing 5 primitive BNs: ComputerWorking, Audio working, Projecting, Studying and Sitting.

We assumed that the service robot would move from place to place and the objects are detected randomly, and recorded the values and hit rates to predict the probability of target objects being present in each place. Fig. 4 shows the results.

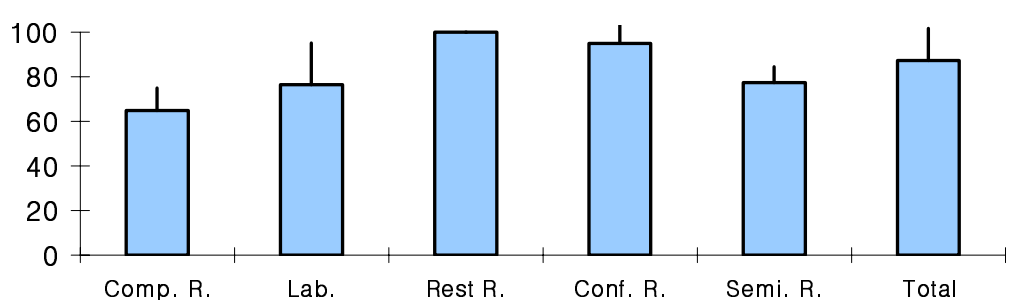

Fig. 4. Accuracy rates at each place

The probabilities of the beam-projector being present in each place were observed under a threshold of $70 \%$ (this value is the best one of our experiments) until the robot was able to find five objects. The overall results shows $87.25 \%$ of accuracy, that is reasonable, but it denoted also that a false-positive error is likely to occur in similar environments (especially Computer room) that contain many objects related to a beam-projector.

\section{Conclusion}

In this paper, we proposed the $\mathrm{BN}$ model for predicting target object when the service robot acts in indoor environment and the method to integrate them for making a large scale $\mathrm{BN}$ for efficient design. For efficient design, we design $\mathrm{BN}$ by combining small BNs for overall structure, and propose binary-cross parameter setting method. The experiments show the proposed BN has good performance.

For the future works, we will devise ensemble method of the results from diverse $\mathrm{BNs}$ for improving the performance, and study a way to make primitive $\mathrm{BN}$ relationship dynamically according to the situation.

\section{Acknowledgement}

This research was supported by the Ministry of Information and Communication, Korea under the Information Technology Research Center support program supervised by the Institute of Information Technology Assessment, IITA-2005-(C10900501-0019). 


\section{References}

1. Murphy, K., et al.: Using the Forest to See the Trees: A Graphical Model Relating Features, Objects, and Scenes. Proc. Neural Info. Proc. System, Vol.16 (2003) 1499-1506

2. Pearl, J.: Probabilistic Reasoning in Intelligent Systems: Networks of Plausible Inference. Morgan Kaufmann, (1988)

3. Laskey, K. B., Mahoney, S. M.: "Network Fragments: Representing Knowledge for Constructing Probabilistic Models." Proc. of 13th Conf. Uncertainty in Artificial Intelligence, (1997) 334-341 